Article

\title{
Chitosan-Modified PLGA Nanoparticles for Control-Released Drug Delivery
}

\author{
Boting Lu, Xikun Lv and Yuan Le *
}

State Key Laboratory of Organic-Inorganic Composites, Beijing University of Chemical Technology, Beijing 100029, China; 2016200044@mail.buct.edu.cn (B.L.); lvmhao@163.com (X.L.)

* Correspondence: leyuan@mail.buct.edu.cn; Tel.: +86-10-64447274

Received: 22 January 2019; Accepted: 9 February 2019; Published: 12 February 2019

\begin{abstract}
Poly (lactic-co-glycolic acid) nanoparticles (PLGA NPs) are well recognized as an ideal drug delivery carrier for their biocompatibility and biodegradability. In order to overcome the disadvantage of drug burst release, chitosan (CS) was used to modify the PLGA nanoparticles. In this work, CS-PLGA nanoparticles with different ratio of CS to PLGA were prepared using high-gravity rotating packed bed (RPB). With the increase of amount of CS, the particle size increased from $132.8 \pm$ $1.5 \mathrm{~nm}$ to $172.7 \pm 3.2 \mathrm{~nm}$, zeta potential increased from $-20.8 \pm 1.1 \mathrm{mV}$ to $25.6 \pm 0.6 \mathrm{mV}$, and drug encapsulation efficiency increased from $65.8 \%$ to $87.1 \%$. The initial burst release of PLGA NPs reduced after being modified by CS, and the cumulative release was $66.9 \%, 41.9 \%, 23.8 \%$, and $14.3 \%$, after $2 \mathrm{~h}$, respectively. The drug release of CS-modified PLGA NPs was faster at $\mathrm{pH} 5.5$ than that at $\mathrm{pH}$ 7.4. The cellular uptake of CS-modified PLGA NPs increased compared with PLGA NPs, while cell viability was reduced. In conclusion, these results indicated that CS-modified, PTX-loaded PLGA NPs have the advantages of sustained drug release and enhanced drug toxicity, suggesting that CS-modified NPs can be used as carriers of anticancer drugs.
\end{abstract}

Keywords: chitosan; PLGA nanoparticles; paclitaxel; drug release

\section{Introduction}

Conventional chemotherapeutic drugs are limited by their toxicity to normal tissues, short circulatory half-life in plasma, limited water solubility, and non-selective therapeutic effects. In the past two decades, nanoparticles (NPs) have attracted considerable interest in the delivery of anticancer drugs and have become an important area of cancer nanotechnology research [1]. Poly (lactic-co-glycolic acid) (PLGA) is an FDA-approved polymer with great biocompatibility and biodegradability. Many researches have demonstrated the great potential of PLGA as a carrier for cancer treatment $[2,3]$. However, one of the major drawbacks of PLGA NPs is that they cannot specifically interact with cells or proteins, which causes an inability to accumulate drugs in target tissues $[4,5]$. Another disadvantage of PLGA NPs is the presence of drug burst release, which could result in side effects [6,7].

To overcome these limitations, chitosan (CS) was used to modify PLGA nanoparticles. CS is a natural cationic polysaccharide with biocompatibility and biodegradability [8]. Chitosan has the capability of forming hydrogen and covalent bonding due to its $-\mathrm{OH}$ and $-\mathrm{NH}_{2}$ groups. The protonation of amino group at low $\mathrm{pH}$, which makes chitosan macromolecule charged positively, leads to the mucosal adhesion of chitosan [9]. Therefore, it is necessary to consider $\mathrm{pH}$ when using CS. Because the $\mathrm{pH}$ of each region of the human body is obviously different [10], it is possible for CS delivery system to achieve targeted delivery of drugs [11]. Sara et al. [12] prepared low molecular weight chitosan-coated PLGA NPs and showed enhanced drug delivery in a weak acid environment. Because of the positive zeta potential, the cellular uptake of CS-modified PLGA NPs could be increased $[13,14]$. However, the suitable amount of CS used for modification has rarely been 
reported. Considering these reports, we were interested in how much the amount of CS modification affects the cytotoxicity and cellular uptake of tumor cells. Knowing this, we could more effectively deliver anticancer drugs.

In this study, PLGA NPs modified with different amount of CS were prepared by the nanoprecipitation. The drug loading, encapsulation efficiency, and in vitro drug release of CS-modified PLGA NPs were studied using paclitaxel (PTX) as a model drug. The effects of CS modification of PLGA NPs on the particle size, zeta potential, and surface morphology were explored. The cytotoxicity and cellular uptake of CS-modified PLGA NPs were studied using MDA-MB-231 cells.

\section{Materials and Methods}

\subsection{Materials}

PLGA (MW = 10,000-20,000, with a lactic acid to glycolic ratio of 75:25) and Nile Red (NR, 98\%) were obtained from Yuanye Bio-Technology (Shanghai, China). Chitosan (MW = $100,000-2,000,000$, viscosity $\leq 800 \mathrm{mPa} \cdot \mathrm{s}$, deacetylation $\geq 80 \%$ ) was obtained from Sinopharm Chemical Reagent Co., Ltd. (Shanghai, China). Poloxamer 188 (F68) and Paclitaxel (PTX) were obtained from Ouhe Technology Co., Ltd. (Beijing, China). Acetone and acetic acid were obtained from Beijing Chemical works (Beijing, China). Acetonitrile was obtained from Aladdin (Shanghai, China). 4',6-diamidino-2-phenylindole (DAPI), dimethyl sulfoxide (DMSO), and 3-(4,5-dimethylthiazol-2-yl)-2,5diphenyl-2-H-tetrazoliumbromide bromide (MTT) were obtained from Sigma-Aldrich (Shanghai, China). All other solvents are of analytical grade and used without further purification.

\subsection{Preparation of PLGA NPS}

Nanoparticles were prepared by nanoprecipitation using high-gravity rotating packed bed (RPB) reactor (Figure 1). PLGA (200 $\mathrm{mg})$ and paclitaxel $(20 \mathrm{mg})$ were added to acetone $(20 \mathrm{~mL})$ to form the organic phase. Poloxamer 188 was added to deionized water $(400 \mathrm{~mL})$ to form the aqueous phase $(0.3 \%$, $\mathrm{w} / \mathrm{v}$ ). Subsequently, the organic phase with a flow rate of $15 \mathrm{~mL} / \mathrm{min}$ and the aqueous phase with a flow rate of $300 \mathrm{~mL} / \mathrm{min}$ were pumped into the RPB reactor. The non-incorporated drug was removed by ultrafiltration centrifugation $(12,000 \mathrm{r} / \mathrm{min}, 15 \mathrm{~min})$. PTX-loaded nanoparticles remained after the supernatant was discarded and the nanoparticles were lyophilized for $48 \mathrm{~h}$ (vacuum freeze-drying machine, LGJ-18S, Songyuan Huaxing, Beijing, China).

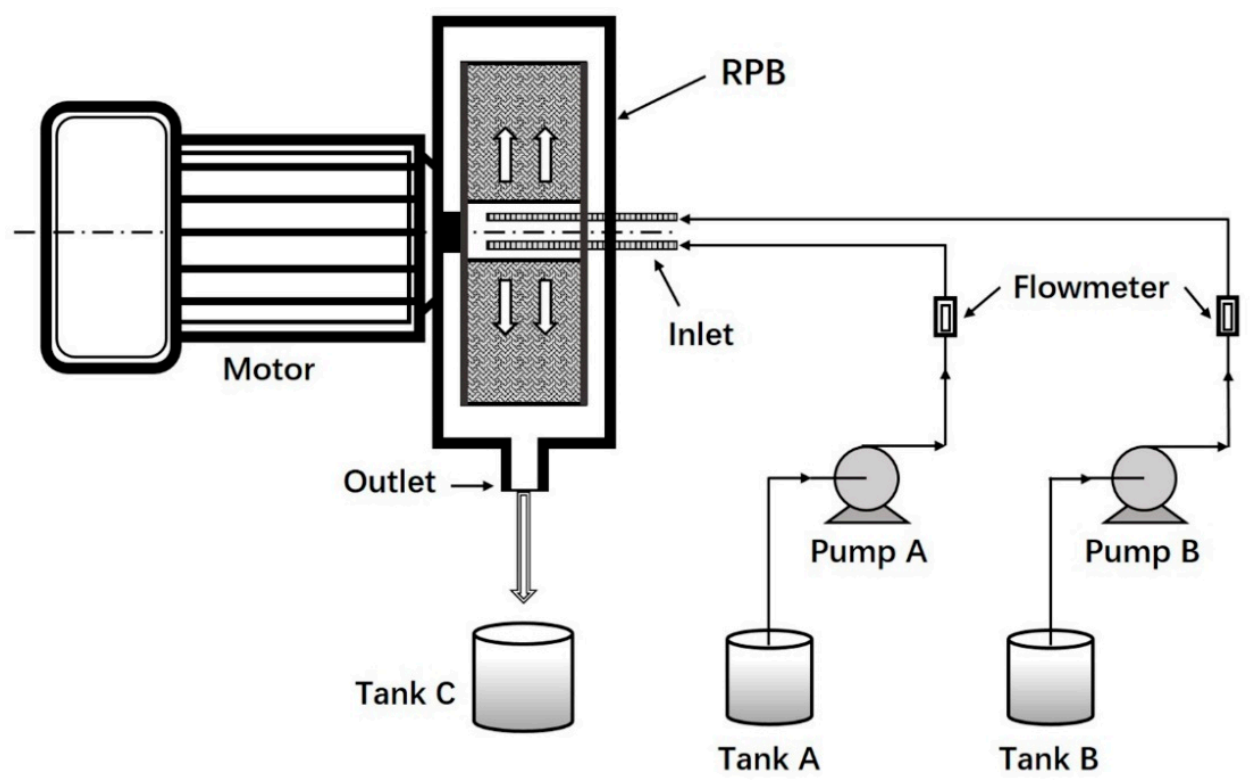

Figure 1. Schematic diagram of experimental setup. 


\subsection{Preparation of the CS-Modified PLGA NPS}

PLGA NPs were modified with CS through electrostatic adherence. Poloxamer $188(0.3 \%, \mathrm{w} / \mathrm{v})$, acetic acid $(4 \mathrm{~mL})$, and CS (40 mg, $80 \mathrm{mg}, 160 \mathrm{mg}$ ) were added to deionized water $(400 \mathrm{~mL})$ to form the aqueous phase. Other steps are consistent with preparation method of PLGA NPs.

\subsection{Characterization of the NPs}

\subsubsection{Particle Size, Zeta Potential, and Surface Morphology of the NPs}

The particle size of the NPs was measured by dynamic light scattering (DLS) on Zetasizer (Nano-ZS90, Malvern Panalytical, Malvern, UK). Additionally, zeta potential of the NPs was also measured on Zetasizer. The freshly prepared NPs were diluted with an appropriate amount of distilled water. Each sample was measured three times. Scanning electron microscopy (SEM, JSM-7800F, JEOL, Tokyo, Japan) was used to observe the surface morphology of NPs.

\subsubsection{TGA, FTIR, and XPS}

The CS content of surface modified NPs was determined by thermal-gravimetric analyzer (TGA, STA-449C, NETZSCH, Selb, Germany). The purified CS or PLGA NPs were placed in alumina crucible and scanned in TGA from 40 to $800{ }^{\circ} \mathrm{C}$ at a rate of $10^{\circ} \mathrm{C} / \mathrm{min}$ under nitrogen flow. The amount of CS in PLGA NPs was determined from the weight loss.

Fourier transform infrared spectroscopy (FTIR, VERTEX 70v, BRUKER, Karlsruhe, Germany) was used to detect the NPs. The dry nanoparticles were weighed at about $2 \mathrm{mg}$ and mixed with the dry potassium bromide $(\mathrm{KBr})$ by grinding. A uniform sheet was made by a tablet press. With air as the background, the wavenumber range was set to $400-4000 \mathrm{~cm}^{-1}$, and the resolution was set to $2 \mathrm{~cm}^{-1}$.

X-ray photoelectron spectroscopy (XPS, ESCALAB 250, THERMO VG, Waltham, MA, USA) was used to determine the surface element composition of nanoparticles. X-ray source was monochromatic $\mathrm{Al} \mathrm{Kalph}$ and the power was $150 \mathrm{~W}$. The pass energy was $200 \mathrm{eV}$ for survey and $30 \mathrm{eV}$ for high resolution scans.

\subsubsection{Drug Loading and Encapsulation Efficiency}

The paclitaxel content in nanoparticles was measured by high performance liquid chromatography (HPLC, MK-II, WATERS, Milford, CT, USA). For HPLC analysis, a reverse-phase XBridge ${ }^{\circledR}$ C18 column $(4.6 \mathrm{~mm} \times 150 \mathrm{~mm}$, pore size $5 \mu \mathrm{m}$, WATERS, Milford, CT, USA) was used. The mobile phase was a mixture of acetonitrile and phosphoric acid (50:50, v/v), the flow rate was set to $1 \mathrm{~mL} / \mathrm{min}$, and the column temperature was set to $30^{\circ} \mathrm{C}$. Paclitaxel was quantified by UV detection at $227 \mathrm{~nm}$. A specific amount of lyophilized nanoparticles was dissolved in $2 \mathrm{~mL}$ acetonitrile and then vortexed vigorously for $5 \mathrm{~min}$. Then, the supernatant was collected and filtered with $0.45 \mu \mathrm{m}$ filter. Drug loading (DL) was the percentage of actual mass of drug loaded in NPs to the total mass of NPs, and the drug encapsulation efficiency (EE) was the percentage of the actual mass of drug loaded in NPs and the initial mass used in the preparation of NPs. The formulas for calculating DL and EE are as follows:

$$
\begin{aligned}
& \mathrm{DL}=\frac{M}{M_{n p}} \times 100 \%, \\
& \mathrm{EE}=\frac{M}{M_{i n}} \times 100 \%,
\end{aligned}
$$

in which $M$ is the actual mass of drug loaded in NPs, $M_{n p}$ is the total mass of NPs, and $M_{i n}$ is the initial mass used in the preparation of NPs. 


\subsubsection{In Vitro Drug-Release Studies}

The in vitro drug release from the NPs was determined in a dissolution tester (Vision G2 Elite 8, HANSON, Chatsworth, CA, USA). $20 \mathrm{mg}$ of PTX-loaded NPs was suspended in $500 \mathrm{~mL}$ PBS (pH 7.4 or 5.5) containing $0.5 \%(\mathrm{w} / \mathrm{w})$ Tween 80 as solubilizer. The rotational speed was set to $100 \mathrm{rpm}$, and the temperature was set to $37^{\circ} \mathrm{C}$. Sampling was at specified time intervals, and an equal amount of fresh PBS was added. Each drug release experiment was tested in triplicate in vitro. The content of PTX in PBS was then determined by HPLC analysis.

\subsubsection{MTT Assay}

The cytotoxicity of PTX-loaded NPs was evaluated using the MTT colorimetric assay. The MDA-MB-231 cells were cultured in 96-well plates of Dulbecco's modified eagle medium (DMEM) containing $10 \%$ fetal bovine serum, and the plates were placed in an incubator at $37{ }^{\circ} \mathrm{C}$ in an environment of $5 \%$ carbon dioxide. The cells were cultured with different concentrations of samples. After the specified incubation time, $20 \mu \mathrm{L}$ MTT was added to each well, and another $4 \mathrm{~h}$ was needed to culture the cells. The medium was then removed and $100 \mathrm{~mL} \mathrm{DMSO}$ was added to 96 -well plates. The color intensity was measured on a microplate reader (Multiskan MK3, THERMO, USA) at $570 \mathrm{~nm}$, and the cell viability was presented and expressed as means \pm standard deviation (SD) $(n=5)$.

\subsubsection{Cellular Uptake of the NPs}

The cells were incubated with neil red-loaded NPs in incubator at $37^{\circ} \mathrm{C}$ in an environment of $5 \%$ carbon dioxide. After $4 \mathrm{~h}$ incubation, the nucleus of cells was stained with DAPI and the cells were fixed with $4 \%$ paraformaldehyde at room temperature. The confocal laser scanning microscopy (CLSM, DMi8, LEICA, Wetzlar, Germany) was used to observe the cellular uptake. NR, an organic fluorescent dye, was used to assist in showing the uptake of NPs in tumor cells.

\section{Results and Discussion}

\subsection{Particle Size, Zeta Potential, and Surface Morphology of the NPs}

Particles smaller than $10 \mathrm{~nm}$ were quickly eliminated by renal changes, while those larger than $300 \mathrm{~nm}$ were removed from the blood circulation due to the recognition of reticuloendothelial system (RES) $[15,16]$. Therefore, 10 to $200 \mathrm{~nm}$ was an ideal range for nanoparticles to promote tumor accumulation. In this study, the particle size of NPs ranged from $132.8 \mathrm{~nm}$ to $172.7 \mathrm{~nm}$ (Table 1). The particle size increased with the modification amount of chitosan.

Table 1. Particle size, polydispersity index (PDI), and zeta potential of nanoparticles (NPs).

\begin{tabular}{cccc}
\hline NPs & Particle Size $(\mathbf{n m})$ & PDI & Zeta Potential (mV) \\
\hline PLGA & $132.8 \pm 1.5$ & $0.155 \pm 0.03$ & $-20.8 \pm 1.1$ \\
CS/PLGA $(w / w)=0.2$ & $140.5 \pm 2.4$ & $0.104 \pm 0.02$ & $10.1 \pm 0.9$ \\
CS/PLGA $(w / w)=0.4$ & $154.2 \pm 2.6$ & $0.122 \pm 0.04$ & $21.5 \pm 0.5$ \\
CS/PLGA $(w / w)=0.8$ & $172.7 \pm 3.2$ & $0.144 \pm 0.06$ & $25.6 \pm 0.6$ \\
\hline
\end{tabular}

The zeta potential of unmodified PLGA NPs was negative $(-20.8 \pm 1.1 \mathrm{mV})$, because of the carboxyl end groups of PLGA molecules located on the surfaces of NPs. The zeta potentials of the CS-modified PLGA NPs were positive, as shown in Table 1, indicating that there some of the amino groups of the CS molecule were located on the surfaces of the NPs. Taken together, these results indicated that the CS had been successfully coated on PLGA NPs. The surface charge properties of nanoparticles were related to their stability and cell adhesion properties [17]. Generally, the greater the absolute zeta potential of nanoparticles, the higher the stability in vitro. When the ratio of chitosan to PLGA is more than 0.4, CS-modified PLGA NPs may have higher stability in vitro. In addition, 
CS-modified PLGA NPs could interact with the negatively charged cell membrane through ionic adsorption $[6,12,18]$, which could contribute to higher cellular uptake.

Surface morphology of the NPs was observed as being smoothly spherical in form using SEM. The CS-modified PLGA NPs were 100-150 $\mathrm{nm}$ in size (Figure 2). SEM images of the nanoparticles suggested they were slightly smaller than those measured by DLS measurements (Table 1). This could mean that SEM shows the NPs in a dry state, while DLS method displays the hydrated layers [19]. Due to the interaction between chitosan molecules, it could be seen that there was more obvious adhesion between nanoparticles as the increase of the amount of CS on the surface of nanoparticles. Additionally, the surface of PLGA NPs that was not modified by CS was smoother than the surface of CS-modified PLGA NPs.

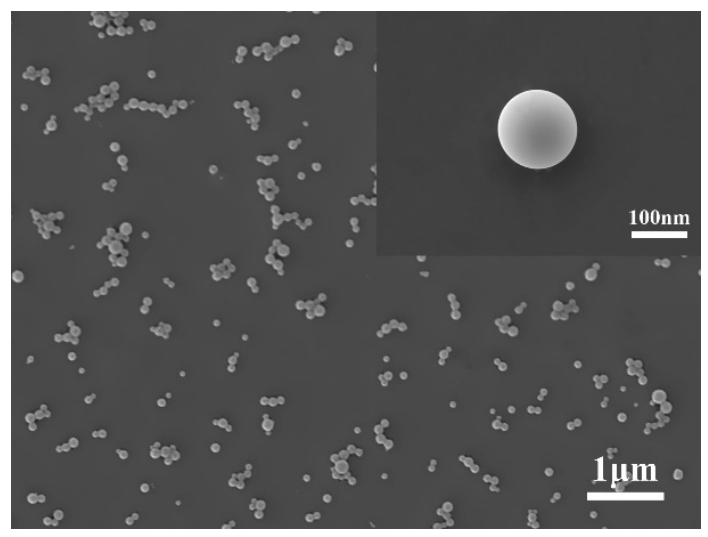

(a)

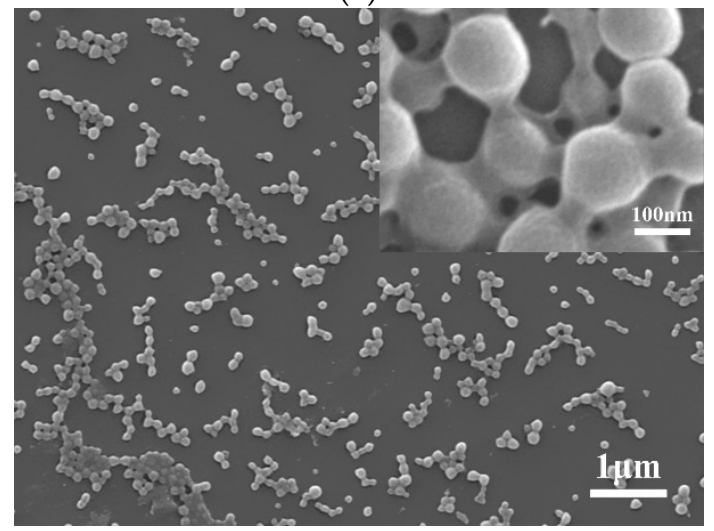

(c)

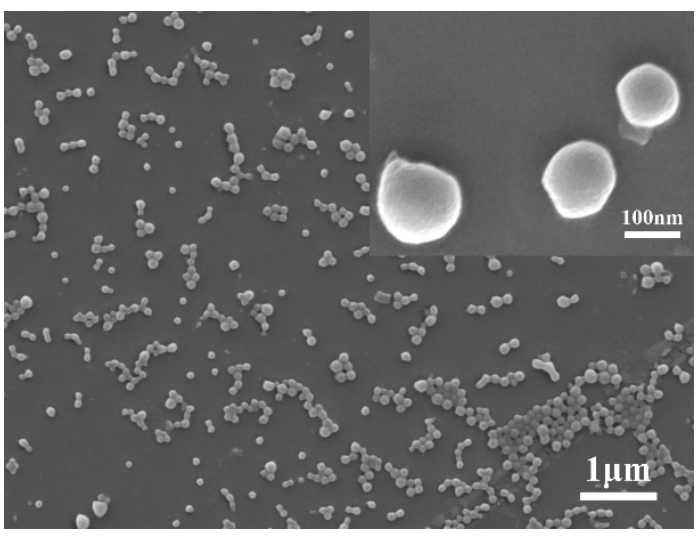

(b)

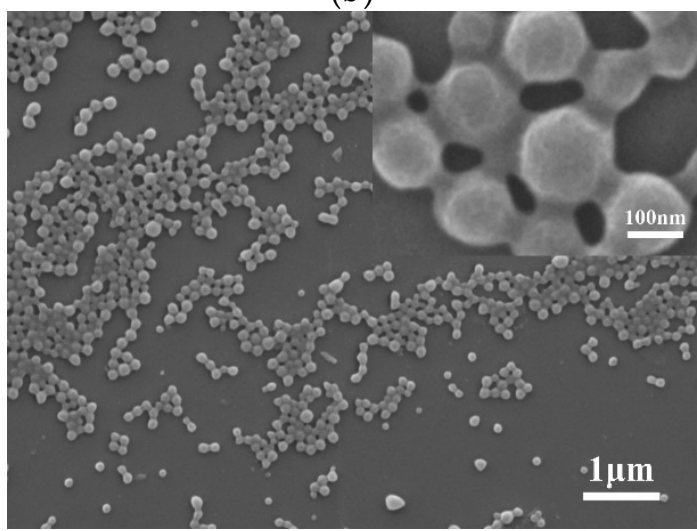

(d)

Figure 2. Scanning electron microscopy (SEM) images of the NPs: (a) poly (lactic-co-glycolic acid) $(\mathrm{PLGA}) ;(\mathbf{b})$ chitosan $(\mathrm{CS}) / \mathrm{PLGA}=0.2 ;(\mathbf{c}) \mathrm{CS} / \mathrm{PLGA}=0.4 ;(\mathbf{d}) \mathrm{CS} / \mathrm{PLGA}=0.8$.

\subsection{TGA, FT-IR, and XPS}

TGA was used to estimate the CS contents on the surfaces of the NPs (Figure 3). The initial temperature of thermal degradation of PLGA was about $315^{\circ} \mathrm{C}$ and degraded completely at $400{ }^{\circ} \mathrm{C}$, with $4.5 \%$ remaining. The weight loss of CS can be divided into two stages; the first stage was the stage of losing water. At the beginning of thermal degradation, CS lost a small amount of weight (5\%), which indicated that CS contained about $5 \%$ free water. The second stage was thermal degradation. CS degraded at about $295^{\circ} \mathrm{C}$, and $34.7 \%$ still remained at $800^{\circ} \mathrm{C}$. The CS-modified PLGA NPs (CS/PLGA $=0.2, \mathrm{CS} / \mathrm{PLGA}=0.4$ and $\mathrm{CS} / \mathrm{PLGA}=0.8)$ remained $9.4 \%, 13.0 \%$ and $17.8 \%$ at $800{ }^{\circ} \mathrm{C}$, respectively. According to the results of thermal analysis, the mass ratios of CS to PLGA were estimated at $19.4 \%$, $39.2 \%$, and $78.7 \%$, respectively, very close to the initio ratio. 


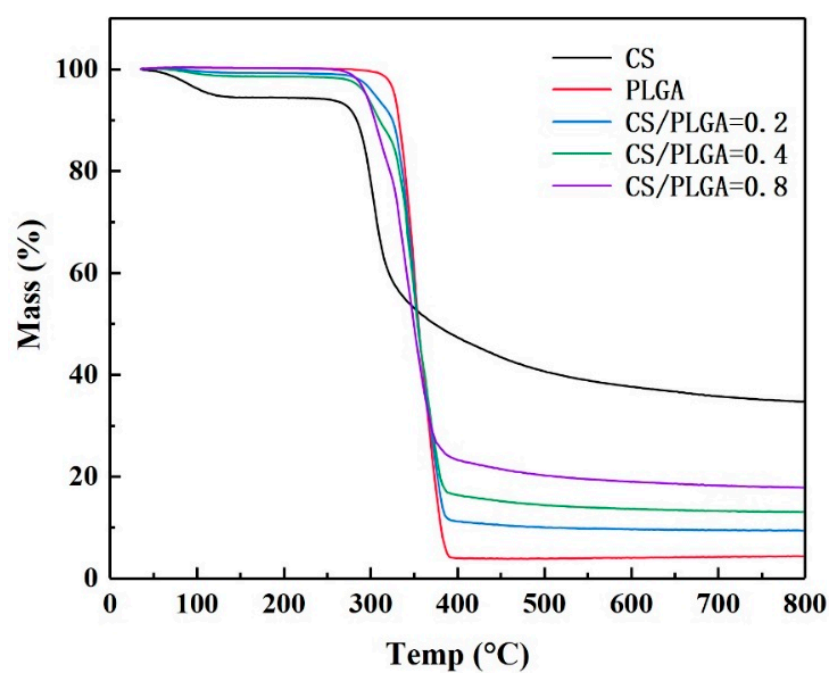

Figure 3. Thermal gravimetric analyzer (TGA) thermograms of NPs.

FT-IR spectra proved the existence of CS on PLGA surface (Figure 4). The FT-IR spectrum of PLGA contained a strong peak at $1094 \mathrm{~cm}^{-1}$ for C-O-C stretching, as well as a peak at $1758 \mathrm{~cm}^{-1}$ for $\mathrm{C}=\mathrm{O}$ stretching. The characteristic band at $3414 \mathrm{~cm}^{-1}$ was ascribed to stretching vibration of $-\mathrm{NH}_{2}$ and $-\mathrm{OH}$ groups in $\mathrm{CS}$. Due to the existence of amide group and $\mathrm{C}-\mathrm{H}$ stretching in $\mathrm{CS}$, strong peaks also appeared at $1567 \mathrm{~cm}^{-1}$. The characteristic peaks of CS were observed in the FT-IR spectrum of CS-modified PLGA nanoparticles, which indicated that CS successfully modified PLGA nanoparticles. It is noteworthy that there are no new peaks in the FTIR spectra of CS-modified PLGA NPs, or in the disappearance of the original peaks. The FTIR spectra of CS-modified PLGA NPs seemed to be the superposition of spectra of PLGA and CS, which indicated that CS modified PLGA nanoparticles by physical adsorption [17].

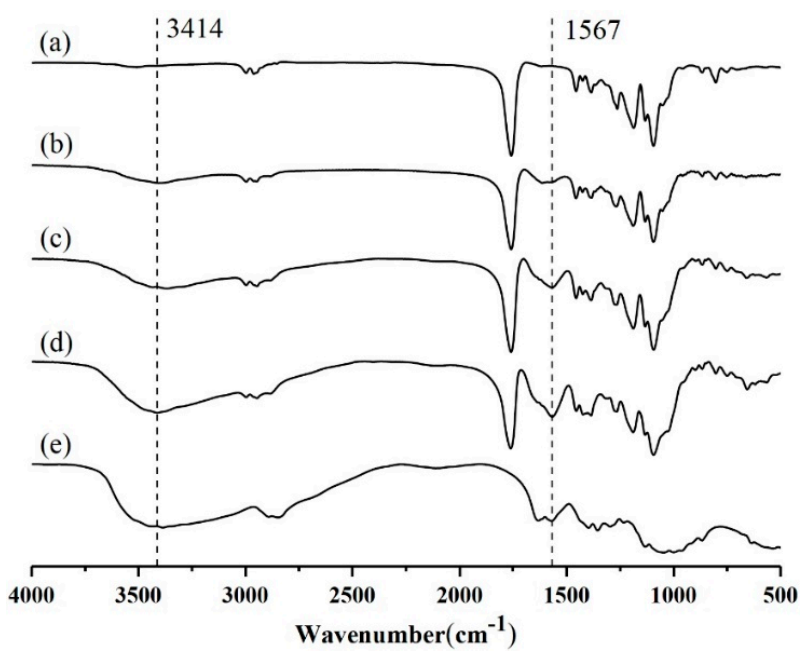

Figure 4. FTIR spectra: (a) PLGA; (b) CS/PLGA = 0.2; (c) CS $/$ PLGA = 0.4; (d) CS/PLGA = 0.8; (e) CS.

XPS was a surface element analysis technology, which could provide quantitative and qualitative information for the existence of various elements on the surface of nanoparticles. Therefore, whether CS was modified on the surface of PLGA NPs could be further confirmed by XPS. The binding energy of $-\mathrm{NH}_{2}$ was about $399 \mathrm{eV}$, while that of $-\mathrm{NHCO}$ was about $402 \mathrm{eV}$, corresponding to amine and amide, respectively [20]. As shown in Figure 5, the CS-modified PLGA NPs show the signal of nitrogen near $400 \mathrm{eV}$, while the PLGA NPs do not observe the signal of nitrogen under the same conditions, which proved that CS has been modified to the surface of nanoparticles. 

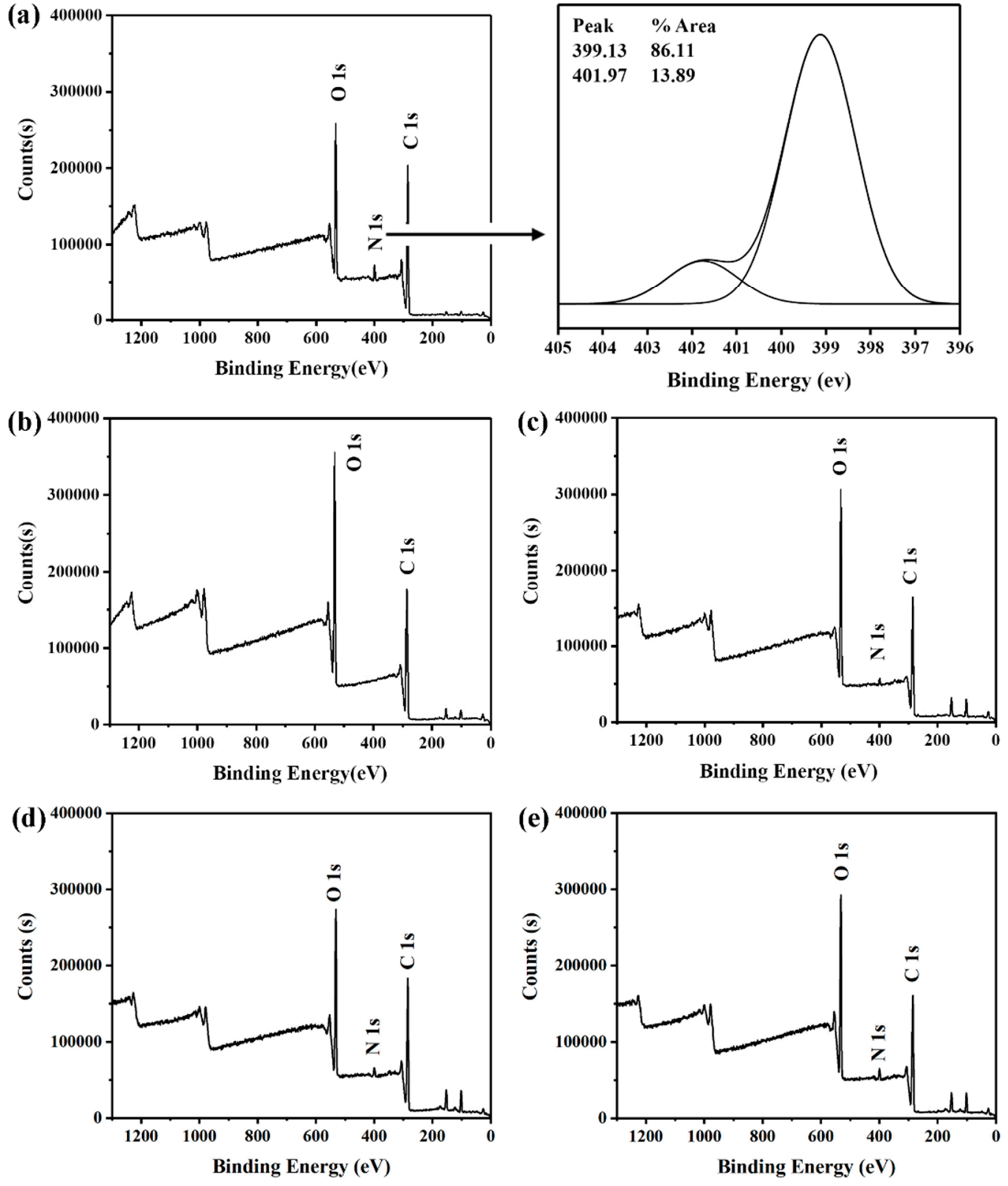

Figure 5. X-ray photoelectron spectroscopy (XPS) spectra: (a) CS; (b) PLGA; (c) CS/PLGA = 0.2; (d) $\mathrm{CS} / \mathrm{PLGA}=0.4 ;(\mathbf{e}) \mathrm{CS} / \mathrm{PLGA}=0.8$.

Furthermore, the N1s signal increased due to the increase of CS. The surface atomic concentration of NPs was listed in Table 2. There was an increase of nitrogen atom concentration as chitosan modification increased. This indicated that there were more amino groups on the surface of CS/PLGA $(\mathrm{w} / \mathrm{w})=0.8$, compared to CS/PLGA $(\mathrm{w} / \mathrm{w})=0.4$ and CS/PLGA $(\mathrm{w} / \mathrm{w})=0.2$. This change also corresponded to the change of surface zeta potential of nanoparticles.

Table 2. Surface atomic concentration of NPs.

\begin{tabular}{cccc}
\hline NPs & C1s & O1s & N1s \\
\hline PLGA & 64.98 & 35.02 & 0 \\
CS & 69.03 & 26.73 & 4.24 \\
CS/PLGA $(w / w)=0.2$ & 65.02 & 32.2 & 2.78 \\
CS/PLGA $(w / w)=0.4$ & 66.29 & 30.19 & 3.52 \\
CS/PLGA $(w / w)=0.8$ & 68.21 & 27.73 & 4.06 \\
\hline
\end{tabular}




\subsection{Drug Loading and Encapsulation Efficiency}

As shown in Table 3, the EE value of the PLGA NPs without modification was $65.8 \%$. Meanwhile, the EE values of CS-modified PLGA NPs (CS/PLGA $=0.2, C S / P L G A=0.4$ and CS/PLGA $=0.8$ ) were $80.5 \%, 85.3 \%$, and $87.1 \%$, respectively. Nanoparticles were prepared by the nanoprecipitation method, which was usually used to encapsulate hydrophobic drugs. Drugs and PLGA dissolve in acetone together. When organic phase contacts water phase, solvent displacement occurs at the interface of two phases. Then, drugs and PLGA deposit into nanoparticles at the interface [21-23]. The formation of nanoparticles occurs at the interface between two phases, and some drug molecules inevitably diffuse into the aqueous phase, resulting in low encapsulation efficiency. This difference in EE values was attributed to the fact that CS can effectively reduce PTX leakage from NPs. Moreover, CS could effectively adsorb PTX because CS was positively charged and PTX was negatively charged. However, with the change of modification amount of CS, the trend of DL was different from that of EE. Although the modification of CS improved the efficiency of drug loading, the total mass of NPs increased. As a result, the DL value decreased. Overall, the modification of CS still had a positive effect.

Table 3. Drug Loading Efficiency and Encapsulation Efficiency of NPs.

\begin{tabular}{ccc}
\hline NPs & DL (\%) & EE (\%) \\
\hline PLGA & 5.07 & 65.8 \\
CS/PLGA (w/w)=0.2 & 6.42 & 80.5 \\
CS/PLGA (w/w) $=0.4$ & 5.69 & 85.3 \\
CS/PLGA $(w / w)=0.8$ & 4.59 & 87.1 \\
\hline
\end{tabular}

\subsection{In Vitro Drug-Release Behavior of NPS}

One of the objectives of this study was to explore the effect of chitosan modification on drug release behavior of nanoparticles. The PBS at $\mathrm{pH} 7.4$ (simulating the $\mathrm{pH}$ of normal human blood) was used to study the release of PTX in NPs. The cumulative release curves were showed in Figure 6. The cumulative release of PTX from PLGA NPs reached $66.9 \%$ after $2 \mathrm{~h}$ because of the presence of burst release. A strong burst release should be avoided, because it can reduce the effect of drug and may cause side effects on the human body [24-27]. Generally speaking, burst release always exists in in vitro drug release of nanoparticles prepared by nanoprecipitation, and it was caused by the adsorption of drugs on the surface of nanoparticles [23]. Charge attraction between drugs and chitosan played an important role in sustained drug release [6]. Due to the adsorption of drugs on chitosan on the surface of nanoparticles, the leakage of PTX was reduced, and the initial release rate of PTX in CS-modified PLGA NPs was significantly slower than that in PLGA NPs. Moreover, the larger the chitosan content in the NPs, the slower the initial release rate of PTX. The cumulative release of PTX from CS-modified PLGA NPs was 41.9\%, 23.8\%, and 14.3\%, after $2 \mathrm{~h}$, respectively. CS-modified PLGA NPs showed more sustained release after the initial rapid release. This result showed that surface modification of CS on PLGA NPs can effectively solve the problem of initial burst release of PLGA NPs and achieve more moderate and sustained release.

The $\mathrm{pH}$-responsive nanoparticles can release drugs rapidly in the tumor site and slowly or even not at all in the peripheral circulation [28-30]. The $\mathrm{pH}$-responsive release of nanoparticles was investigated by two different buffer solutions $(\mathrm{pH}=5.5$ and $\mathrm{pH}=7.4)$ in vitro. As shown in Figure 7a, the release patterns of PLGA NPs at two different $\mathrm{pHs}$ were similar, which indicated that the release of PTX-loaded PLGA NPs was pH-independent. However, the release rate of PTX in CS-modified PLGA NPs was very low at pH 7.4, and only $41.9 \%, 23.8 \%$, and $14.3 \%$ PTX was released within $2 \mathrm{~h}$, respectively. Meanwhile, $60.9 \%, 51.7 \%$, and $40.9 \%$ PTX was released after $2 \mathrm{~h}$ at $\mathrm{pH} 5.5$, respectively, indicating that CS-modified NPs had $\mathrm{pH}$-responsive properties. The pKa of Chitosan is to 6.3 due to the free amino group $[14,20,31]$. When the $\mathrm{pH}$ is higher than 6.3 , these amino groups deprotonate and uncharge, thus forming an insoluble biopolymer shell. However, when the $\mathrm{pH}$ is less than 6.3 , these 
amino groups are protonated and the solubility of chitosan in acidic $\mathrm{pH}$ increased [31]. Due to the higher solubility of CS at pH 5.5, CS on the surface of NPs could no longer effectively slow down the release rate of PTX.

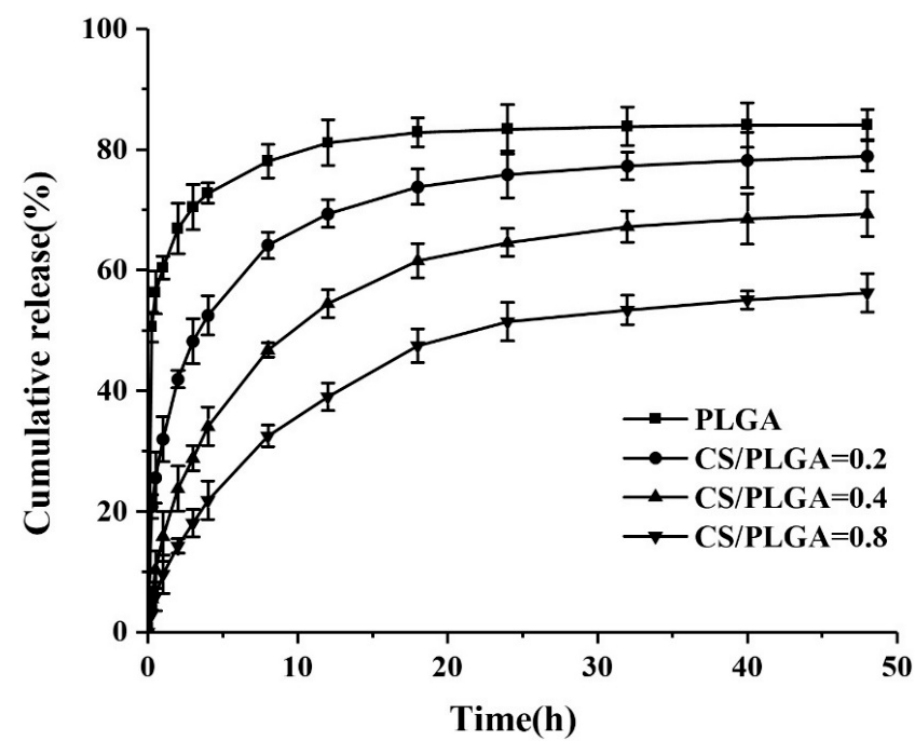

Figure 6. In vitro release of paclitaxel (PTX) from NPs at $\mathrm{pH}=7.4$.

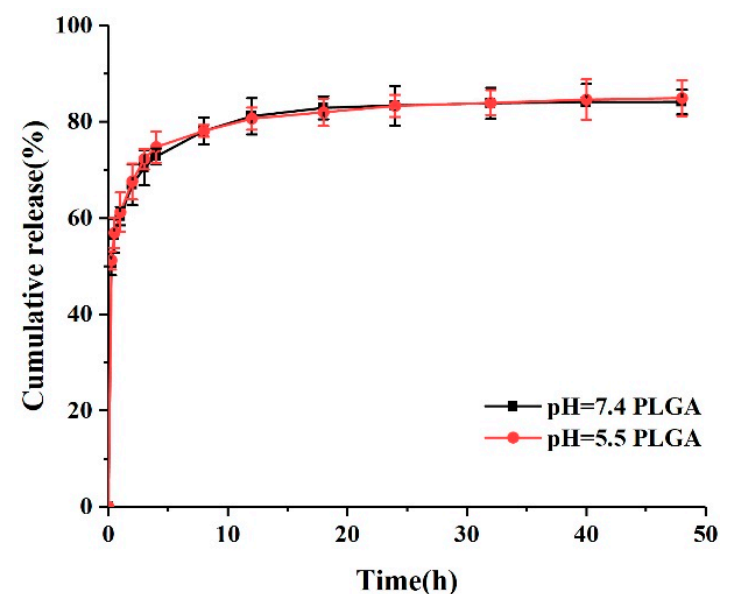

(a)

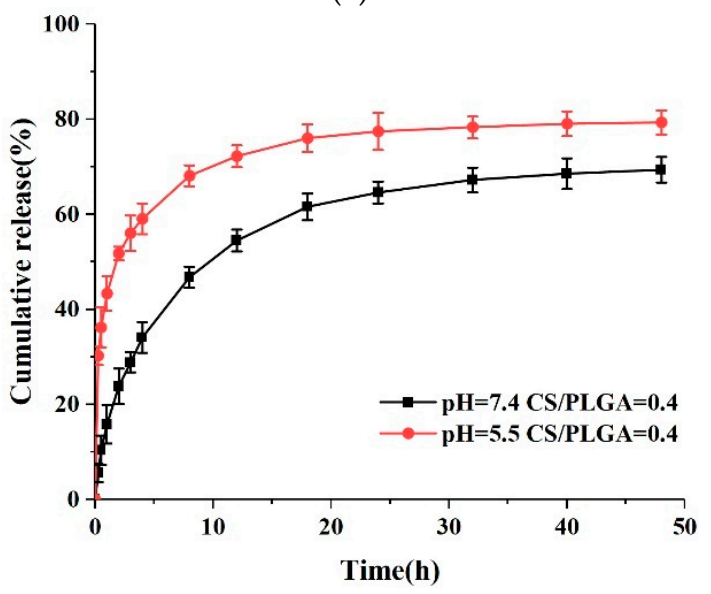

(c)

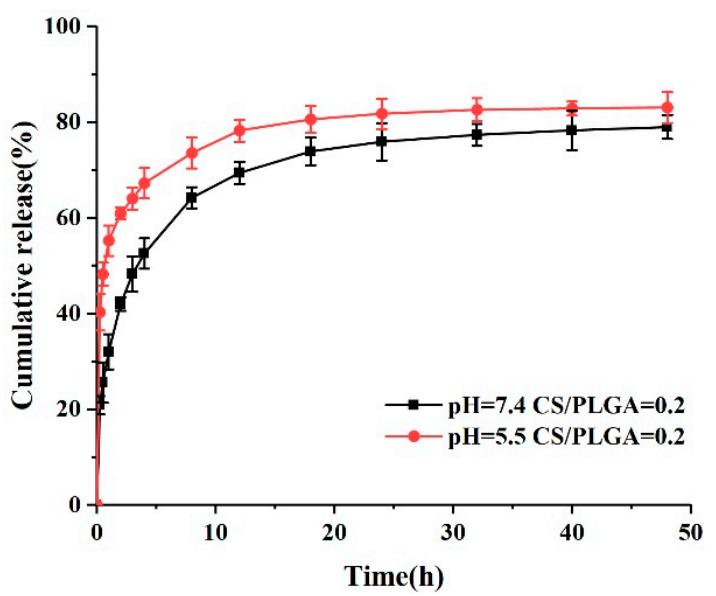

(b)

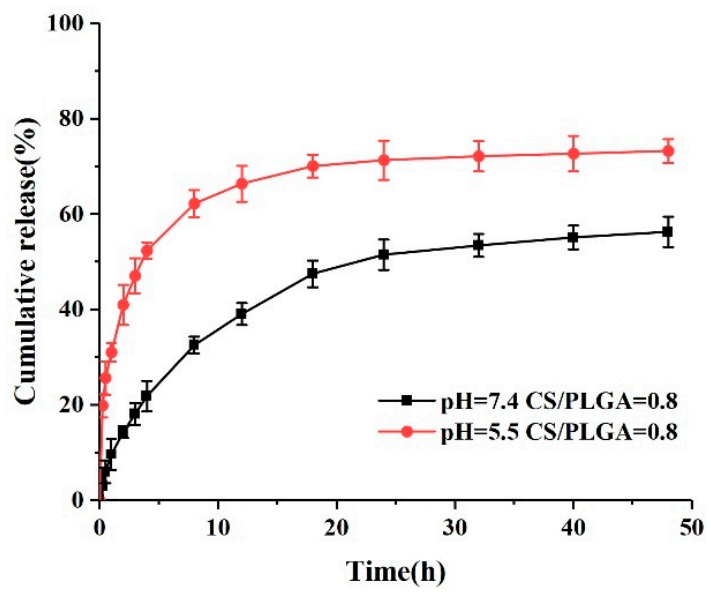

(d)

Figure 7. In vitro release of PTX from NPs at $\mathrm{pH} 7.4$ and $\mathrm{pH}$ 5.5: (a) PLGA; (b) CS/PLGA = 0.2; (c) $\mathrm{CS} / \mathrm{PLGA}=0.4 ;$ and $(\mathbf{d}) \mathrm{CS} / \mathrm{PLGA}=0.8$. 


\subsection{MTT Assay}

Although the safety of PLGA has been widely recognized due to its biocompatibility and biodegradability, it is possible that CS-modified PLGA NPs were cytotoxic. Blank NPs were also evaluated. Figure 8 showed the effect of PTX-loaded NPs and blank NPs on the proliferation of MDA-MB-231 cells. According to the results, the cell viability of blank NPs increased slightly with the increased amounts of CS coating on the nanoparticle, indicating that CS were not cytotoxic and CS even had a positive effect on the safety of PLGA NPs. Compared with free PTX, the PTX-loaded NPs could reduce the cell viability of MDA-MB-231 cells, which means NPs had a higher level of cytotoxicity. According to previous reports [32], drug-loaded nanoparticles can enter tumor cells through endocytosis and then release the drug into cells. This approach could overcome the resistance mechanism of tumor cells by avoiding the efflux of P-glycoprotein. Meanwhile, the cell viability decreased significantly with the increase of chitosan modification, suggesting that chitosan modification may contribute to the cellular uptake of PLGA NPs.

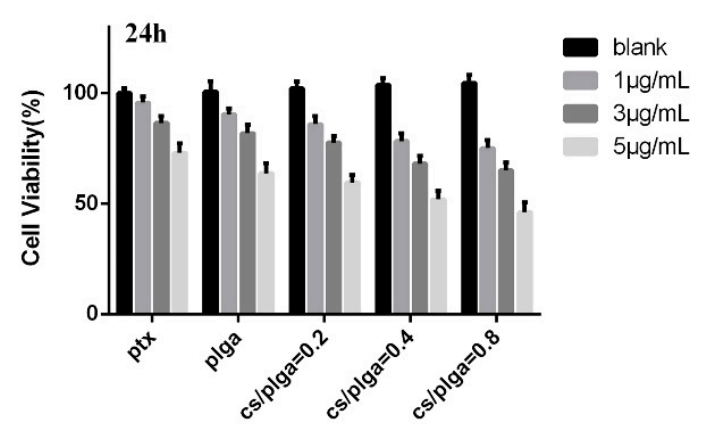

(a)

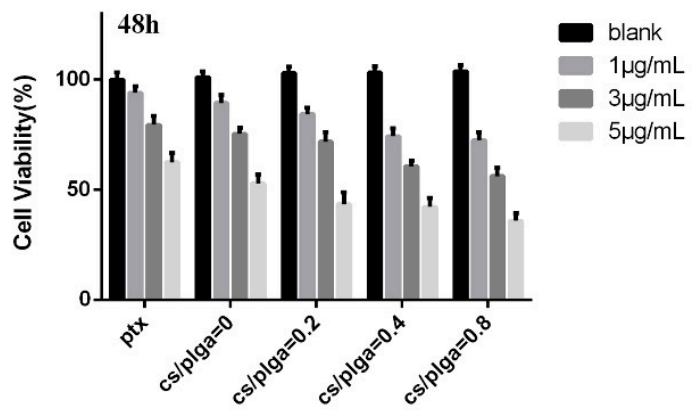

(b)

Figure 8. Cell viability of MDA-MB-231 cells: (a) $24 \mathrm{~h}$ and (b) $48 \mathrm{~h}$.

\subsection{Cellular Uptake of the NPS}

CLSM was used to show the uptake of NPs by tumor cells. NR-loaded NPs were prepared by the same method as PTX-loaded NPs. NR was a water-insoluble organic fluorescent dye, and existed in the form of large particles in aqueous solution. As shown in Figure 9, the free NR could not enter MDA-MB-231 cells, and NR-loaded NPs could effectively enter MDA-MB-231 cells through endocytosis. This result demonstrated the advantage of NPs in transporting drugs into cells.

Compared with NR-loaded PLGA NPs, CS-modified PLGA NPs show higher cellular uptake. Positive-charged CS-based NPs can promote endocytosis by interacting with negatively charged cell membranes [33]. Therefore, CS-modified PLGA NPs can enhance drug uptake by tumor cells [34]. It is worth noting that CS-modified PLGA NPs entered the nucleus of MDA-MB-231 cells while PLGA NPs did not. Previous studies $[35,36]$ stated clearly that PLGA nanoparticles could enter the nucleus with the help of surface-modified cationic polymers. According to Figure 9, the amount of modification of CS was an important factor affecting the entry of NPs into the nucleus. Meanwhile, it needed to be large enough to ensure that NPs could enter the nucleus effectively. These results show the potentiality of CS-modified PLGA NPs as gene carrier. 


\section{Bright Field}

(a)

(b)

(c)

(d)

(e)

(f)
DAPI
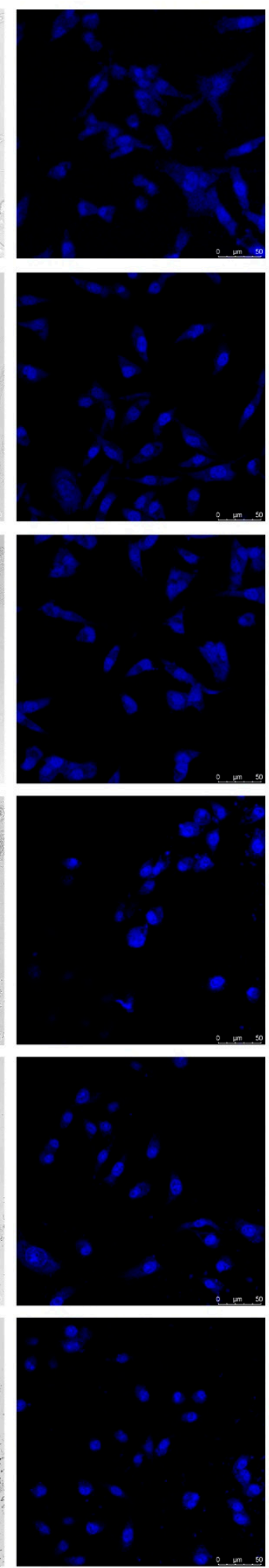

NR
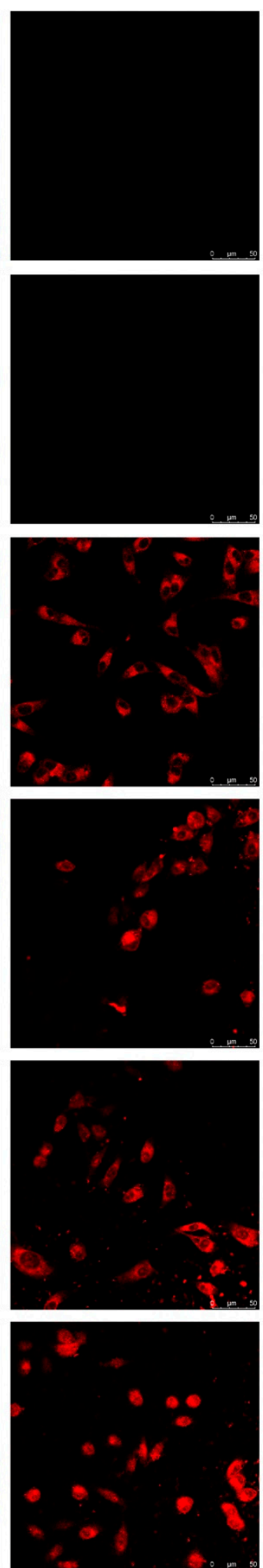

Overlay
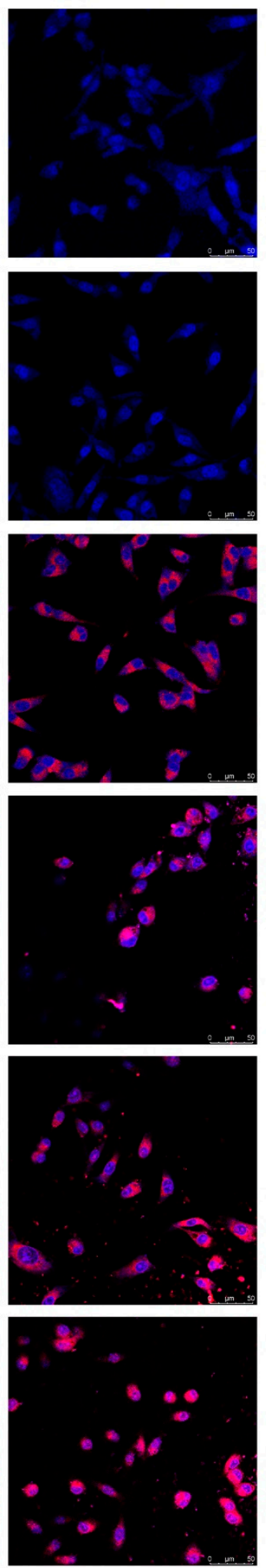

Figure 9. Confocal laser scanning microscopy (CLSM) images: (a) control; (b) raw NR; (c) PLGA; (d) $\mathrm{CS} / \mathrm{PLGA}=0.2 ;(\mathbf{e}) \mathrm{CS} / \mathrm{PLGA}=0.4 ;$ and $(\mathbf{f}) \mathrm{CS} / \mathrm{PLGA}=0.8$. 


\section{Conclusions}

In this study, PLGA NPs were modified by different amount of CS. The particle size of nanoparticles was in an appropriate range, and changed from $132.8 \mathrm{~nm}$ to $172.7 \mathrm{~nm}$. The zeta potential of PLGA NPs became positive after being modified by CS. The efficiency of drug loading increased with the increase of the amount of CS. The initial burst release of PLGA NPs was effectively reduced after being modified by CS, and presented a more sustained release. CS-modified PLGA NPs were $\mathrm{pH}$-responsive and released faster at $\mathrm{pH} 5.5$, which contributed to faster drug release in tumor tissue. Meanwhile, CS-modified PLGA NPs showed higher levels of cytotoxicity and cellular uptake in MDA-MB-231 cells than PTX-loaded PLGA NPs and free PTX. These results suggested that the CS-modified PLGA NPs have potential as an antitumor drug carrier. The amount of CS modified on the surface of PLGA NPs could affect the properties of PLGA NPs, and suitable carriers could be designed to deal with this problem.

Author Contributions: B.L. conceived and designed the experiments; B.L. and X.L. performed the experiments; B.L. analyzed the data and wrote the paper; and Y.L. revised the paper.

Acknowledgments: This work was supported by National Key Basic Research Program of China (2015CB932100) and National Natural Science Foundation of China (21622601).

Conflicts of Interest: The authors declare no conflict of interest.

\section{References}

1. Brigger, I.; Dubernet, C.; Couvreur, P. Nanoparticles in cancer therapy and diagnosis. Adv. Drug Deliv. Rev. 2012, 64, 24-36. [CrossRef]

2. Danhier, F.; Ansorena, E.; Silva, J.M.; Coco, R.; Le Breton, A.; Preat, V. PLGA-based nanoparticles: An overview of biomedical applications. J. Control. Release 2012, 161, 505-522. [CrossRef] [PubMed]

3. Varypataki, E.M.; Silva, A.L.; Barnier-Quer, C.; Collin, N.; Ossendorp, F.; Jiskoot, W. Synthetic long peptide-based vaccine formulations for induction of cell mediated immunity: A comparative study of cationic liposomes and PLGA nanoparticles. J. Control. Release 2016, 226, 98-106. [CrossRef] [PubMed]

4. Wu, J.; Deng, C.; Meng, F.; Zhang, J.; Sun, H.; Zhong, Z. Hyaluronic acid coated PLGA nanoparticulate docetaxel effectively targets and suppresses orthotopic human lung cancer. J. Control. Release 2017, 259, 76-82. [CrossRef] [PubMed]

5. El-Hammadi, M.M.; Delgado, A.V.; Melguizo, C.; Prados, J.C.; Arias, J.L. Folic acid-decorated and PEGylated PLGA nanoparticles for improving the antitumour activity of 5-fluorouracil. Int. J. Pharm. 2017, 516, 61-70. [CrossRef] [PubMed]

6. Chen, H.; Xie, L.Q.; Qin, J.; Jia, Y.; Cai, X.; Nan, W.; Yang, W.; Lv, F.; Zhang, Q.Q. Surface modification of PLGA nanoparticles with biotinylated chitosan for the sustained in vitro release and the enhanced cytotoxicity of epirubicin. Colloids Surf. B Biointerfaces 2016, 138, 1-9. [CrossRef]

7. Wang, F.; Yuan, J.; Zhang, Q.; Yang, S.; Jiang, S.; Huang, C. PTX-loaded three-layer PLGA/CS/ALG nanoparticle based on layer-by-layer method for cancer therapy. J. Biomater. Sci. Polym. Ed. 2018, 29, 1566-1578. [CrossRef]

8. Ali, A.; Ahmed, S. A review on chitosan and its nanocomposites in drug delivery. Int. J. Biol. Macromol. 2018, 109, 273-286. [CrossRef]

9. Ways, T.M.M.; Lau, W.M.; Khutoryanskiy, V.V. Chitosan and Its Derivatives for Application in Mucoadhesive Drug Delivery Systems. Polymers 2018, 10, 267. [CrossRef]

10. Engin, K.; Leeper, D.B.; Cater, J.R.; Thistlethwaite, A.J.; Tupchong, L.; McFarlane, J.D. Extracellular pH distribution in human tumours. Int. J. Hyperthermia 1995, 11, 211-216. [CrossRef]

11. Huang, G.; Liu, Y.; Chen, L. Chitosan and its derivatives as vehicles for drug delivery. Drug Deliv. 2017, 24, 108-113. [CrossRef] [PubMed]

12. Abouelmagd, S.A.; Ku, Y.J.; Yeo, Y. Low molecular weight chitosan-coated polymeric nanoparticles for sustained and pH-sensitive delivery of paclitaxel. J. Drug Target. 2015, 23, 725-735. [CrossRef] [PubMed]

13. Chen, H.L.; Wang, Y.X. Chitosan Surface-Modified PLGA Nanoparticles: Preparation, Characterization, and Evaluation of their In Vitro Drug-Release Behaviors and Cytotoxicities. Curr. Nanosci. 2014, 10, $255-262$. [CrossRef] 
14. Taghavi, S.; Ramezani, M.; Alibolandi, M.; Abnous, K.; Taghdisi, S.M. Chitosan-modified PLGA nanoparticles tagged with 5TR1 aptamer for in vivo tumor-targeted drug delivery. Cancer Lett. 2017, 400, 1-8. [CrossRef] [PubMed]

15. Fox, M.E.; Szoka, F.C.; Frechet, J.M. Soluble polymer carriers for the treatment of cancer: the importance of molecular architecture. Acc. Chem. Res. 2009, 42, 1141-1151. [CrossRef] [PubMed]

16. Kobayashi, H.; Watanabe, R.; Choyke, P.L. Improving Conventional Enhanced Permeability and Retention (EPR) Effects; What Is the Appropriate Target? Theranostics 2014, 4, 81-89. [CrossRef] [PubMed]

17. Chen, H.; Yang, W.; Chen, H.; Liu, L.; Gao, F.; Yang, X.; Jiang, Q.; Zhang, Q.; Wang, Y. Surface modification of mitoxantrone-loaded PLGA nanospheres with chitosan. Colloids Surf. B Biointerfaces 2009, 73, 212-218. [CrossRef]

18. Shariatinia, Z. Pharmaceutical applications of chitosan. Adv. Colloid Interface Sci. 2019, 263, 131-194. [CrossRef]

19. Vu-Quang, H.; Vinding, M.S.; Xia, D.; Nielsen, T.; Ullisch, M.G.; Dong, M.; Nielsen, N.C.; Kjems, J. Chitosan-coated poly (lactic-co-glycolic acid) perfluorooctyl bromide nanoparticles for cell labeling in (19) F magnetic resonance imaging. Carbohydr. Polym. 2016, 136, 936-944. [CrossRef]

20. Lawrie, G.; Keen, I.; Drew, B.; Chandler-Temple, A.; Rintoul, L.; Fredericks, P.; Grondahl, L. Interactions between alginate and chitosan biopolymers characterized using FTIR and XPS. Biomacromolecules 2007, 8, 2533-2541. [CrossRef]

21. Fessi, H.; Puisieux, F.; Devissaguet, J.P.; Ammoury, N.; Benita, S. Nanocapsule formation by interfacial polymer deposition following solvent displacement. Int. J. Pharm. 1989, 55, R1-R4. [CrossRef]

22. Martinez Rivas, C.J.; Tarhini, M.; Badri, W.; Miladi, K.; Greige-Gerges, H.; Nazari, Q.A.; Galindo Rodriguez, S.A.; Roman, R.A.; Fessi, H.; Elaissari, A. Nanoprecipitation process: From encapsulation to drug delivery. Int. J. Pharm. 2017, 532, 66-81. [CrossRef] [PubMed]

23. Almoustafa, H.A.; Alshawsh, M.A.; Chik, Z. Technical aspects of preparing PEG-PLGA nanoparticles as carrier for chemotherapeutic agents by nanoprecipitation method. Int. J. Pharm. 2017, 533, 275-284. [CrossRef] [PubMed]

24. Rodrigues de Azevedo, C.; von Stosch, M.; Costa, M.S.; Ramos, A.M.; Cardoso, M.M.; Danhier, F.; Preat, V.; Oliveira, R. Modeling of the burst release from PLGA micro- and nanoparticles as function of physicochemical parameters and formulation characteristics. Int. J. Pharm. 2017, 532, 229-240. [CrossRef] [PubMed]

25. Din, F.U.; Kim, D.W.; Choi, J.Y.; Thapa, R.K.; Mustapha, O.; Kim, D.S.; Oh, Y.K.; Ku, S.K.; Youn, Y.S.; Oh, K.T.; et al. Irinotecan-loaded double-reversible thermogel with improved antitumor efficacy without initial burst effect and toxicity for intramuscular administration. Acta Biomater. 2017, 54, 239-248. [CrossRef] [PubMed]

26. Li, J.; Sun, L.; Liu, Y.; Yao, H.; Jiang, S.; YunzhuPu; Li, Y.; Zhang, Y. To reduce premature drug release while ensuring burst intracellular drug release of solid lipid nanoparticle-based drug delivery system with clathrin modification. Nanomedicine 2019, 15, 108-118. [CrossRef] [PubMed]

27. Wu, H.Y.; Hu, Z.H.; Jin, T. Sustained-release microspheres of amifostine for improved radio-protection, patient compliance, and reduced side effects. Drug Deliv. 2016, 23, 3704-3711. [CrossRef]

28. Kocak, G.; Tuncer, C.; Bütün, V. pH-Responsive polymers. Polym. Chem. 2017, 8, 144-176. [CrossRef]

29. Vivek, R.; Nipun Babu, V.; Thangam, R.; Subramanian, K.S.; Kannan, S. pH-responsive drug delivery of chitosan nanoparticles as Tamoxifen carriers for effective anti-tumor activity in breast cancer cells. Colloids Surf. B Biointerfaces 2013, 111, 117-123. [CrossRef]

30. Yilmaz, E.; Yalinca, Z.; Yahya, K.; Sirotina, U. pH responsive graft copolymers of chitosan. Int. J. Biol. Macromol. 2016, 90, 68-74. [CrossRef]

31. Khanal, S.; Adhikari, U.; Rijal, N.P.; Bhattarai, S.R.; Sankar, J.; Bhattarai, N. pH-Responsive PLGA Nanoparticle for Controlled Payload Delivery of Diclofenac Sodium. J. Funct. Biomater. 2016, 7, 21. [CrossRef] [PubMed]

32. Cartiera, M.S.; Johnson, K.M.; Rajendran, V.; Caplan, M.J.; Saltzman, W.M. The uptake and intracellular fate of PLGA nanoparticles in epithelial cells. Biomaterials 2009, 30, 2790-2798. [CrossRef] [PubMed]

33. Termsarasab, U.; Cho, H.J.; Kim, D.H.; Chong, S.; Chung, S.J.; Shim, C.K.; Moon, H.T.; Kim, D.D. Chitosan oligosaccharide-arachidic acid-based nanoparticles for anti-cancer drug delivery. Int. J. Pharm. 2013, 441, 373-380. [CrossRef] [PubMed] 
34. Chronopoulou, L.; Massimi, M.; Giardi, M.F.; Cametti, C.; Devirgiliis, L.C.; Dentini, M.; Palocci, C. Chitosan-coated PLGA nanoparticles: a sustained drug release strategy for cell cultures. Colloids Surf. B Biointerfaces 2013, 103, 310-317. [CrossRef] [PubMed]

35. Kasturi, S.P.; Sachaphibulkij, K.; Roy, K. Covalent conjugation of polyethyleneimine on biodegradable microparticles for delivery of plasmid DNA vaccines. Biomaterials 2005, 26, 6375-6385. [CrossRef] [PubMed]

36. Zhang, D.; Kong, Y.Y.; Sun, J.H.; Huo, S.J.; Zhou, M.; Gui, Y.L.; Mu, X.; Chen, H.; Yu, S.Q.; Xu, Q. Co-delivery nanoparticles with characteristics of intracellular precision release drugs for overcoming multidrug resistance. Int. J. Nanomed. 2017, 12, 2081-2108. [CrossRef] [PubMed]

(C) 2019 by the authors. Licensee MDPI, Basel, Switzerland. This article is an open access article distributed under the terms and conditions of the Creative Commons Attribution (CC BY) license (http:/ / creativecommons.org/licenses/by/4.0/). 